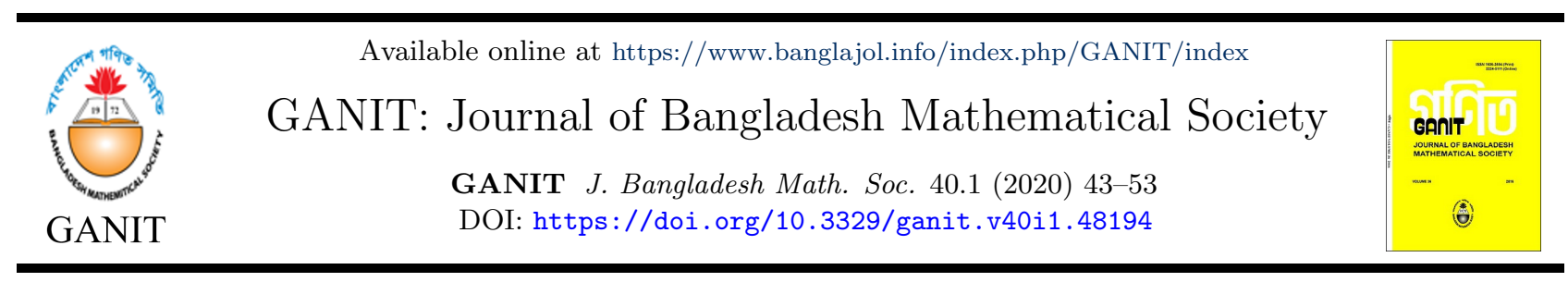

\title{
On the Convergence of Newton-like Method for Variational Inclusions under Pseudo-Lipschitz Mapping
}

\author{
Mst. Zamilla Khaton ${ }^{\mathrm{a}}$, M. H. Rashid*b, and M. I. Hossain ${ }^{\mathrm{c}}$ \\ a,b,c Department of Mathematics, University of Rajshahi, Rajshahi-6205, Bangladesh \\ a Department of Mathematics, BCS General Education, DSHE, Dhaka, Bangladesh
}

\begin{abstract}
In the present paper, we study a Newton-like method for solving the variational inclusion defined by the sums of a Fréchet differentiable function, divided difference admissible function and a set-valued mapping with closed graph. Under some suitable assumptions on the Fréchet derivative of the differentiable function and divided difference admissible function, we establish the existence of any sequence generated by the Newton-like method and prove that the sequence generated by this method converges linearly and superlinearly to a solution of the variational inclusion. Specifically, when the Fréchet derivative of the differentiable function is continuous, Lipschitz continuous, divided difference admissible function admits first order divided difference and the setvalued mapping is pseudo-Lipschitz continuous, we show the linear and superlinear convergence of the method.
\end{abstract}

(c) 2020 Published by Bangladesh Mathematical Society Received: 22 March, 2020

Accepted: 09 July, 2020

Keywords: Set-valued mapping; Pseudo-Lipschitz continuity; Local convergence; Superlinear convergence; Divided difference.

AMS Subject Classification 2010: 33F05 - 46N40 - 65T50 - 41A50.

\section{Introduction}

Let $X$ and $Y$ be real or complex Banach spaces. In this study, we are concerned with the problem of finding a solution $x^{*} \in \Omega$ satisfying the variational inclusion of the form

$$
0 \in f(x)+g(x)+F(x),
$$

where $f: \Omega \subseteq X \rightarrow Y$ is a single-valued function which is Fréchet differentiable in a neighborhood $\Omega$ of a solution $x^{*}$ of (1.1), $g: \Omega \subseteq X \rightarrow Y$ is differentiable at $x^{*}$ but may not differentiable in $\Omega$ and $F: X \rightrightarrows Y$ is a set-valued mapping with closed graph.

Let us remark that the variational inclusion type (1.1), were introduced by Robinson [25,26], is an abstract model for various problems and it has been explored as a general tool for describing, analyzing, and solving different problems in a unified manner. These type of inclusion problems have been studied extensively; see for

\footnotetext{
*Corresponding author: M. H. Rashid, E-mail address: harun_math@ru.ac.bd
} 
examples [6, 8-10,12-15,18]. There have many applications of variational inclusion (1.1) in systems of inequalities, variational inequalities, linear and nonlinear complementarity problems, systems of nonlinear equations, equilibrium problems, etc.; see for example [8].

When $F=0,(1.1)$ is reduced to the classical problem of solving systems of nonlinear equations: $0 \in$ $f(x)+g(x)$. Cătinas [2] proposed the following method for solving $0 \in f(x)+g(x)$ by using the combination of Newton's method with the secants method when $f$ is differentiable and $g$ is a continuous function admitting first and second order divided differences:

$$
0 \in f\left(x_{k}\right)+g\left(x_{k}\right)+\left(\mathcal{D} f\left(x_{k}\right)+\left[x_{k-1}, x_{k} ; g\right]\right)\left(x_{k+1}-x_{k}\right), k=1,2, \ldots,
$$

where $\mathcal{D} f(x)$ denotes the Fréchet derivative of $f$ at $x$ and $[x, y ; g]$ is the first order divided difference of $g$ on the points $x$ and $y$.

For solving (1.1), Jean-Alexis and Pietrus [11] presented the following method:

$$
0 \in f\left(x_{k}\right)+g\left(x_{k}\right)+\left(\mathcal{D} f\left(x_{k}\right)+\left[2 x_{k+1}-x_{k}, x_{k} ; g\right]\right)\left(x_{k+1}-x_{k}\right)+F\left(x_{k+1}\right) .
$$

They proved that this sequence generated by (1.2) converges superlinearly by considering that $\mathcal{D} f$ and the first order divided difference of $g$ are $p$-Hölder continuous around a solution $x^{*}$ and that $(f+g+F)^{-1}$ is pseudoLipschitz around $\left(0, x^{*}\right)$ with $F$ having closed graph. In recent time, Rashid et al. [24] have been presented the improvement of the result corresponding one in Jean-Alexis and Piétrus [11] and show that if $\mathcal{D} f$ and the first order divided difference of $g$ are $p$-Hölder continuous at a solution $x^{*}$, then the method (1.2) converges superlinearly. A vast number of iterative procedures have been introduced and studied for solving (1.1); see for details in [19-23].

When $g=0$, the inclusion (1.1) reduce to a variational inclusion of the form

$$
0 \in f(x)+F(x) .
$$

Various iterative methods have been studied for solving (1.3). Dontchev [3] established a quadratically convergent Newton-type method under a pseudo-Lipschitz property for set-valued mapping when $\mathcal{D} f$ is Lipschitz on a neighborhood of a solution $x^{*}$ of $(1.3)$ and subsequently he [5] proved the stability of this method. When $\mathcal{D} f$ is Hölder on a neighborhood of $x^{*}$, Pietrus [17] obtained superlinear convergence by following Dontchev's method and later he [16] proved the stability of this method in this mild differentiability context. In the case $g=0$, Geoffroy et al. [9] considered a second degree Taylor polynomial expansion of $f$ under suitable first and second order differentiability assumptions and showed that the existence of a sequence cubically converging to the solution of (1.1). But we cannot apply the above methods, because the lack of regularity of $g$. To carry out our objective, we propose a combination of Newton's method with the secant's one. When the single-valued functions involved in (1.1) is differentiable, Newton-type method can be considered to solve this variational inclusion, such an approach has been used in many contributions to this subject; see for example [1,3,4,7]. To solve the problem (1.1), Geoffroy and Pietrus [11] associated in the following iterative method

$$
0 \in f\left(x_{k}\right)+g\left(x_{k}\right)+\left(\mathcal{D} f\left(x_{k}\right)+\left[x_{k-1}, x_{k} ; g\right]\right)\left(x_{k+1}-x_{k}\right)+F\left(x_{k+1}\right)
$$

and studied this method by using the assumptions that $\mathcal{D} f$ and the second-order divided difference of $g$ are Lipschitz continuous around a solution $x^{*}$. They proved that the sequence generated by (1.4) converges superlinearly.

The aim of this study is to extend the result given in [11] by using the concept of the first-order divided difference of $g$ and $\mathcal{D} f$ is continuous and Lipschitz continuous and then we prove the existence of a sequence generated by the method (1.4) and show the linear and superlinear convergence of the method for solving the variational inclusion (1.1).

This work is organized as follows: In Section 2, we recall few preliminary results that will be used in the next sections. In Section 3, we make some fundamental assumptions on $\mathcal{D} f$ and $g$ and prove the existence of a sequence $\left\{x_{k}\right\}$ satisfying (1.4). Moreover, we show that the sequence $\left\{x_{k}\right\}$ generated by the method (1.4) converges linearly and superlinearly to the solution $x^{*}$ of (1.1). In Section 4, we will give conclusion of the major results obtained in this study.

\section{Notations and Preliminary Results}

Let $X$ and $Y$ be real or complex Banach spaces. Suppose that $f: X \rightarrow Y$ is a Fréchet differentiable function and $F: X \rightrightarrows Y$ is a set-valued mapping with closed graph. The Graph of $F$ is defined by the set 
$\operatorname{gph} F:=\{(x, y) \in X \times Y: y \in F(x)\}$ and the inverse of $F$ is defined by $F^{-1}(y):=\{x \in X: y \in F(x)\}$. All the norms are denoted by $\|\cdot\|$. The closed ball centered at $x$ with radius $r>0$ is denoted by $\mathbb{B}_{r}(x)$ and $\mathcal{L}(\mathcal{X}, \mathcal{Y})$ stands for the set of all bounded linear operators from $X$ to $Y$. Let $A, B \subseteq X$. The distance from a point $x$ to a set $A$ is defined by $\operatorname{dist}(x, A):=\inf \{\|x-a\|: a \in A\}$ for each $x \in X$ while the excess $e$ from the set $A$ to a set $B$ is defined by $e(B, A):=\sup \{\operatorname{dist}(x, A): x \in B\}$.

The following definitions of continuity, Lipschitz continuity and Hölder continuity are taken from the book [2].

Definition 1. A map $f: \Omega \subseteq X \rightarrow Y$ is said to be continuous at $\bar{x} \in \Omega$ if for every $\epsilon>0$, there exist a $\delta>0$ such that

$$
\|f(x)-f(\bar{x})\|<\epsilon, \text { for all } x \in \Omega \text {, for which }\|x-\bar{x}\|<\delta .
$$

Definition 2. A map $f: \Omega \subseteq X \rightarrow Y$ is said to be Lipschitz continuous if there exists constant $0<c<1$ such that

$$
\|f(x)-f(y)\| \leq c\|x-y\|, \text { for all } x \text { and } y \text { in the domain of } f .
$$

The following definitions of linear convergence and quadratic convergence are taken from the book [11].

Definition 3. Let $\left\{x_{n}\right\}$ be a sequence which converges to the number $\bar{x}$. Then the sequence $\left\{x_{n}\right\}$ is said to be converges linearly to $\bar{x}$, if there exists a number $0<c<1$ such that

$$
\left\|x_{n+1}-\bar{x}\right\| \leq c\left\|x_{n}-\bar{x}\right\|
$$

Definition 4. Let $\left\{x_{n}\right\}$ be a sequence which converges to the number $\bar{x}$. Then the sequence $\left\{x_{n}\right\}$ is said to be converges quadratically to $\bar{x}$, if there exists a number $0<c<1$ such that

$$
\left\|x_{n+1}-\bar{x}\right\| \leq c\left\|x_{n}-\bar{x}\right\|^{2} .
$$

The following definition is taken from Dontchev and Hager [7].

Definition 5. Let $F: X \rightrightarrows Y$ be a set-valued mapping. Then $F$ is said to be pseudo-Lipschitz around $\left(x_{0}, y_{0}\right) \in \operatorname{gph} F$ with constant $M>0$ if there exist $\alpha>0$ and $\beta>0$ such that the following inequality holds:

$$
e\left(F\left(x_{1}\right) \cap \mathbb{B}_{\beta}\left(y_{0}\right), F\left(x_{2}\right)\right) \leq M\left\|x_{1}-x_{2}\right\| \quad \text { for any } \quad x_{1}, x_{2} \in \mathbb{B}_{\alpha}\left(x_{0}\right) .
$$

When $F$ is single-valued, this corresponds to the usual concept of Lipschitz continuity. The definition of Lipschitz continuity is equivalent to the definition of Aubin continuity, which is given below:

A set-valued map $\Gamma: Y \rightrightarrows X$ is Aubin continuous at $\left(y_{0}, x_{0}\right) \in g p h \Gamma$ with positive constants $\alpha, \beta$ and $M$ if for every $y_{1}, y_{2} \in B_{\beta}\left(y_{0}\right)$ and for every $x_{1} \in \Gamma\left(y_{1}\right) \cap \mathbb{B}_{\alpha}\left(x_{0}\right)$, there exists an $x_{2} \in \Gamma\left(y_{2}\right)$ such that

$$
\left\|x_{1}-x_{2}\right\| \leq M\left\|y_{1}-y_{2}\right\| .
$$

The constant $\mathrm{M}$ is called the modulus of Aubin continuity.

The definition of the first order divided difference is collected from [24]:

Definition 6. An operator, belonging to the space $\mathcal{L}(\mathcal{X}, \mathcal{Y})$ denoted by $\left[x_{0}, y_{0} ; g\right]$, is called the first order divided difference of the operator $g: X \rightarrow Y$ on the points $x_{0}, y_{0} \in X$ if both of the following properties hold:

(a) $\left[x_{0}, y_{0} ; g\right]\left(y_{0}-x_{0}\right)=g\left(y_{0}\right)-g\left(x_{0}\right)$ for $x_{0} \neq y_{0}$;

(b) If $g$ is Fréchet differentiable at $x_{0} \in X$ then $\left[x_{0}, x_{0} ; g\right]=g^{\prime}\left(x_{0}\right)$.

The following Lemma is known as Banach fixed-point theorem, which has been proved by Dontchev and Hagger in [7] . This fixed-point lemma is the vital mechanism to prove the existence of any sequence generated by (1.4).

Lemma 1. Let $\Phi: X \rightrightarrows X$ be a set-valued mapping and let $\eta_{0} \in X, r>0$ and $0<\lambda<1$ be such that

(a) $\operatorname{dist}\left(\eta_{0}, \Phi\left(\eta_{0}\right)\right)<r(1-\lambda)$ and

(b) $e\left(\Phi\left(x_{1}\right) \cap \mathbb{B}_{r}\left(\eta_{0}\right), \Phi\left(x_{2}\right)\right) \leq \lambda\left\|x_{1}-x_{2}\right\| \quad$ for any $x_{1}, x_{2} \in \mathbb{B}_{r}\left(\eta_{0}\right)$.

Then $\Phi$ has a fixed point in $\mathbb{B}_{r}\left(\eta_{0}\right)$, that is, there exists $x \in \mathbb{B}_{r}\left(\eta_{0}\right)$ such that $x \in \Phi(x)$. If $\Phi$ is single-valued, then $x$ is the unique fixed point of $\Phi$ in $\mathbb{B}_{r}\left(\eta_{0}\right)$. 


\section{Convergence Analysis}

This section is devoted to study the existence and the convergence of any sequence generated by the method (1.4) for the variational inclusion (1.1). Let $f: X \rightarrow Y$ be a single valued continuous function, $g: X \rightarrow Y$ admits first order divided difference and $F: X \rightrightarrows Y$ be a set-valued mapping. Let $x^{*}$ be a solution of (1.1). Let $x \in X$ and define a set valued mapping $Q_{x^{*}}: X \rightrightarrows Y$ by

$$
Q_{x^{*}}(\cdot):=f\left(x^{*}\right)+g(\cdot)+\mathcal{D} f\left(x^{*}\right)\left(\cdot-x^{*}\right)+F(\cdot) .
$$

Consider the following assumptions:

(A0) F has closed graph;

(A1) $f$ is Fréchet differentiable in a neighborhood of $x^{*}$;

(A2) $g$ is differentiable at $x^{*}$;

(A3) The set valued map $Q_{x^{*}}^{-1}$ is $M$-pseudo-Lipschitz around $\left(0, x^{*}\right)$.

Define a single valued function $Z_{k}: X \rightarrow Y$, for $k \in \mathbb{N}$ and $x_{k} \in X$, by

$$
Z_{k}(x)(=) f\left(x^{*}\right)+g(x)+\mathcal{D} f\left(x^{*}\right)\left(x-x^{*}\right)-f\left(x_{k}\right)-g\left(x_{k}\right)-\left(\mathcal{D} f\left(x_{k}\right)+\left[x_{k-1}, x_{k} ; g\right]\right)\left(x-x_{k}\right),
$$

Also, define a set valued mapping $\Phi_{k}: X \rightrightarrows X$ by

$$
\Phi_{k}(x)=Q_{x^{*}}^{-1}\left[Z_{k}(x)\right]
$$

\subsection{Linear Convergence}

This subsection is devoted to study linear convergence result of the Newton-like method (1.4). To do this we will take the following assumptions:

(A4) $\mathcal{D} f$ is continuous in a neighbourhood of $x^{*}$ with constant $\epsilon>0$ i.e. for every $\epsilon>0$, there exists $\delta>0$ such that

$$
\|\mathcal{D} f(x)-\mathcal{D} f(y)\|<\epsilon, \quad \text { whenever }\|x-y\| \leq \delta
$$

(A5) $g$ admits first order divided difference i.e. there exists $\kappa>0$ such that, for all $x, y, x^{\prime}, y^{\prime} \in \Omega$,

$$
\left\|[x, y ; g]-\left[x^{\prime}, y^{\prime} ; g\right]\right\| \leq \kappa\left(\left\|x-x^{\prime}\right\|+\left\|y-y^{\prime}\right\|\right) \quad \text { with } x^{\prime} \neq x, y^{\prime} \neq y
$$

Let $M, \epsilon$ and $\kappa$ be defined in (A3), (A4) and (A5) respectively satisfying the relation $14 M(\epsilon+4 \kappa)<3$.

$$
\text { Set } C:=\frac{7 M(\epsilon+4 \kappa)}{3} .
$$

This together with above inequality implies that $C<\frac{1}{2}$.

Lemma 2. Let $x^{*}$ be a solution of (1.1). Suppose that assumptions (A0)-(A5) are hold.. Let $C$ be defined by (3.4). Then for every such $C$, there exists $\delta>0$ such that for every distinct starting points $x_{0}, x_{1} \in B_{\delta}\left(x^{*}\right)$, there exists a sequence $\left\{x_{2}\right\}$, defined by

$$
0 \in f\left(x_{1}\right)+g\left(x_{1}\right)+\left(\mathcal{D} f\left(x_{1}\right)+\left[x_{0}, x_{1} ; g\right]\right)\left(x_{2}-x_{1}\right)+F\left(x_{2}\right),
$$

and the map $\Phi_{1}$ has a fixed point $x_{2}$ in $B_{\delta}\left(x^{*}\right)$, which satisfies

$$
\left\|x_{2}-x^{*}\right\| \leq C\left\|x_{1}-x^{*}\right\| .
$$


Proof. The assumption (A3) implies that the mapping $Q_{x^{*}}^{-1}$ is $M$-pseudo-Lipschitz around $\left(0, x^{*}\right)$. Hence there exists $r_{x^{*}}>0$ and $r_{0}>0$ such that

$$
e\left(Q_{x}^{*-1}\left(y_{1}\right) \cap \mathbb{B}_{r_{x^{*}}}\left(x^{*}\right), Q_{x^{*}}^{-1}\left(y_{2}\right)\right) \leq M\left\|y_{1}-y_{2}\right\| \text { for any } y_{1}, y_{2} \in \mathbb{B}_{r_{0}}(0) .
$$

Let $\delta>0$ be such that

$$
\delta \leq \max \left\{r_{x^{*}}, \frac{r_{0}}{3 \epsilon+8 \kappa}, \frac{4-7 M \epsilon}{28 M \kappa}, 1\right\} .
$$

Fix $x_{0}, x_{1} \in \mathbb{B}_{\delta}\left(x^{*}\right)$ such that $x_{0} \neq x_{1} \neq x^{*}$, and define

$$
r_{x_{2}}=C\left\|x_{1}-x^{*}\right\|
$$

Since $C<\frac{1}{2}$ from (3.4) and for $x_{0}, x_{1} \in \mathbb{B}_{\delta}\left(x^{*}\right)$, we have

$$
r_{x_{2}}=C\left\|x_{1}-x^{*}\right\| \leq c \cdot \delta \leq \frac{\delta}{2}
$$

This shows that $r_{x_{2}} \leq \delta \leq r_{x^{*}}$.

We will apply Lemma 1 to the map $\Phi_{1}$ with $\eta_{0}:=x^{*}$ and $r:=r_{x_{2}}$ and $\lambda:=\frac{4}{7}$ to conclude that there exists a fixed point $x_{2} \in \mathbb{B}_{r_{x_{2}}}\left(x^{*}\right)$ such that $x_{2} \in \Phi_{1}\left(x_{2}\right)$, that is, $Z_{1}\left(x_{2}\right) \in Q_{x^{*}}{ }^{-1}\left(x_{2}\right)$, which implies that

$$
0 \in f\left(x_{1}\right)+g\left(x_{1}\right)+\left(\mathcal{D} f\left(x_{1}\right)+\left[x_{0}, x_{1} ; g\right]\right)\left(x_{2}-x_{1}\right)+F\left(x_{2}\right),
$$

i.e. (3.5) holds. Furthermore, $x_{2} \in \mathbb{B}_{r_{x_{2}}}\left(x^{*}\right) \subseteq \mathbb{B}_{\delta}\left(x^{*}\right)$ and so

$$
\left\|x_{2}-x^{*}\right\| \leq r_{x_{2}}=C\left\|x_{1}-x^{*}\right\|,
$$

i.e. (3.6) holds. Thus, to complete the proof, it is sufficient to show that Lemma 1 is applicable for the map $\Phi_{1}$ with $\eta_{0}:=x^{*}$ and $r:=r_{x_{2}}$ and $\lambda:=\frac{4}{7}$. To do this, it remains to prove that both assertions (a) and (b) of Lemma 1 hold. It is obvious that $x^{*} \in Q_{x^{*}}^{-1}(0) \cap \mathbb{B}_{r_{x_{2}}}\left(x^{*}\right)$. According to the definition of the excess $e$, we have

$$
\operatorname{dist}\left(x^{*}, \Phi_{1}\left(x^{*}\right)\right) \leq e\left(Q_{x^{*}}^{-1}(0) \cap \mathbb{B}_{r_{x_{2}}}\left(x^{*}\right), \Phi_{1}\left(x^{*}\right)\right)
$$

Moreover, for all $x_{0}, x_{1} \in \mathbb{B}_{r_{x_{2}}}\left(x^{*}\right)$ such that $x_{0}, x_{1}$ and $x^{*}$ are distinct, we have from (3.2) that

$$
\begin{aligned}
\left\|Z_{1}\left(x^{*}\right)\right\| & =\| f\left(x^{*}\right)+g\left(x^{*}\right)-f\left(x_{1}\right)-g\left(x_{1}\right)-\left(\mathcal{D} f\left(x_{1}\right)+\left[x_{0}, x_{1} ; g\right]\right)\left(x^{*}-x_{1}\right) \\
& \leq\left\|f\left(x^{*}\right)-f\left(x_{1}\right)-\mathcal{D} f\left(x_{1}\right)\left(x^{*}-x_{1}\right)\right\|+\left\|g\left(x^{*}\right)-g\left(x_{1}\right)-\left[x_{0}, x_{1} ; g\right]\left(x^{*}-x_{1}\right)\right\| \\
& \leq\left\|f\left(x^{*}\right)-f\left(x_{1}\right)-\mathcal{D} f\left(x_{1}\right)\left(x^{*}-x_{1}\right)\right\|+\left\|\left[x_{1}, x^{*} ; g\right]\left(x^{*}-x_{1}\right)-\left[x_{0}, x_{1} ; g\right]\left(x^{*}-x_{1}\right)\right\|
\end{aligned}
$$

[By using definition (6)].

$$
\leq\left\|f\left(x^{*}\right)-f\left(x_{1}\right)-\mathcal{D} f\left(x_{1}\right)\left(x^{*}-x_{1}\right)\right\|+\left\|\left(\left[x_{1}, x^{*} ; g\right]-\left[x_{0}, x_{1} ; g\right]\right)\left(x^{*}-x_{1}\right)\right\|
$$

Since $f\left(x^{*}\right)-f\left(x_{1}\right)-\mathcal{D} f\left(x_{1}\right)\left(x^{*}-x_{1}\right)=\int_{0}^{1}\left[\mathcal{D} f\left(x_{1}+t\left(x^{*}-x_{1}\right)\right)-\mathcal{D} f\left(x_{1}\right)\right]\left(x^{*}-x_{1}\right) d t$, we have that

$$
\begin{aligned}
\left\|Z_{1}\left(x^{*}\right)\right\| \leq & \int_{0}^{1}\left\|\left[\mathcal{D} f\left(x_{1}+t\left(x^{*}-x_{1}\right)\right)-\mathcal{D} f\left(x_{1}\right)\right]\left(x^{*}-x_{1}\right)\right\| d t+\left\|\left[x_{1}, x^{*} ; g\right]-\left[x_{0}, x_{1} ; g\right]\left(x^{*}-x_{1}\right)\right\| \\
\leq & \epsilon \int_{0}^{1}\left\|x^{*}-x_{1}\right\| d t+\kappa\left(\left\|x_{1}-x_{0}\right\|+\left\|x^{*}-x_{0}\right\|\right)\left\|x^{*}-x_{1}\right\| \\
& \quad[\text { By using assumptions (A4)\& (A5)] } \\
= & \epsilon\left\|x^{*}-x_{1}\right\| \int_{0}^{1} d t+\kappa\left(\left\|x_{1}-x_{0}\right\|+\left\|x^{*}-x_{0}\right\|\right)\left\|x^{*}-x_{1}\right\| \\
\leq & \epsilon\left\|x^{*}-x_{1}\right\|+\kappa\left(\left\|x_{1}-x^{*}+x^{*}-x_{0}\right\|+\left\|x^{*}-x_{1}\right\|\right)\left\|x^{*}-x_{1}\right\| \\
= & \epsilon \delta+\kappa(2 \delta+\delta) \delta=\epsilon \delta+3 \kappa \delta^{2} \\
\leq & \epsilon \delta+3 \kappa \delta=(\epsilon+3 \kappa) \delta<r_{0}, \quad \text { by (3.8). }
\end{aligned}
$$


This together with (3.7) and (3.10) (with $y_{1}=0$ and $y_{2}=Z_{1}\left(x^{*}\right)$ ) implies that

$$
\begin{aligned}
\operatorname{dist}\left(x^{*}, \Phi_{1}\left(x^{*}\right)\right) & \leq M\left\|y_{1}-y_{2}\right\| \leq M\left\|Z_{1}\left(x^{*}\right)\right\| \\
& \leq M\left(\epsilon\left\|x^{*}-x_{1}\right\|+\kappa\left(\left\|x_{1}-x_{0}\right\|+\left\|x^{*}-x_{0}\right\|\right)\left\|x^{*}-x_{1}\right\|\right), \quad \text { by using }(3.11) \\
& \leq M\left(\epsilon+2 \kappa\left\|x_{1}-x_{0}\right\|\right)\left\|x_{1}-x^{*}\right\| \\
& \leq M(\epsilon+4 \kappa \delta)\left\|x_{1}-x^{*}\right\| \\
& \leq M(\epsilon+4 \kappa)\left\|x_{1}-x^{*}\right\|, \quad \text { Since } \delta \leq 1 \quad \text { (by using 3.8) } \\
& \leq\left(1-\frac{4}{7}\right) r_{x_{2}}=r(1-\lambda)
\end{aligned}
$$

Hence assertion (a) of Lemma 1 is satisfied.

Now, we show that assertion (b) of Lemma 1 is also satisfied. Let $x \in \mathbb{B}_{\delta}\left(x^{*}\right)$. Then

$$
\begin{aligned}
\left\|Z_{1}(x)\right\|= & \left\|f\left(x^{*}\right)+g(x)-\mathcal{D} f\left(x^{*}\right)\left(x^{*}-x\right)-f\left(x_{1}\right)-g\left(x_{1}\right)-\left(\mathcal{D} f\left(x_{1}\right)+\left[x_{0}, x_{1} ; g\right]\right)\left(x-x_{1}\right)\right\| \\
= & \| f\left(x^{*}\right)-f(x)+f(x)-f\left(x_{1}\right)-\mathcal{D} f\left(x^{*}\right)\left(x^{*}-x\right)+g(x)-g\left(x_{1}\right) \\
& \quad-\left(\mathcal{D} f\left(x_{1}\right)\left(x-x_{1}\right)-\left[x_{0}, x_{1} ; g\right]\right)\left(x-x_{1}\right) \| \\
\leq & \left\|f\left(x^{*}\right)-f(x)-\mathcal{D} f\left(x^{*}\right)\left(x^{*}-x\right)\right\|+\left\|f(x)-f\left(x_{1}\right)-\mathcal{D} f\left(x_{1}\right)\left(x-x_{1}\right)\right\| \\
& \quad+\left\|g(x)-g\left(x_{1}\right)-\left[x_{0}, x_{1} ; g\right]\left(x-x_{1}\right)\right\| \\
\leq & \epsilon\left\|x-x^{*}\right\|+\epsilon\left\|x-x_{1}\right\|+\left\|\left[x_{1}, x ; g\right]\left(x-x_{1}\right)-\left[x_{0}, x_{1} ; g\right]\left(x-x_{1}\right)\right\| \\
+ & \epsilon\left\|x-x^{*}\right\|+\epsilon\left\|x-x_{1}\right\|+\left\|\left[x_{1}, x ; g\right]-\left[x_{0}, x_{1} ; g\right]\right\|\left\|x-x_{1}\right\| \\
\leq & \epsilon\left\|x-x^{*}\right\|+\epsilon\left\|x-x_{1}\right\|+\kappa\left(\left\|x_{1}-x_{0}\right\|+\left\|x-x_{1}\right\|\right)\left\|x-x_{1}\right\| \\
\leq & \epsilon \delta+2 \epsilon \delta+\kappa(2 \delta+2 \delta) 2 \delta=3 \epsilon \delta+8 \kappa \delta^{2} \\
\leq & 3 \epsilon \delta+8 \kappa \delta, \quad \text { since } \delta \leq 1 \\
= & (3 \epsilon+8 \kappa) \delta<r_{0} \quad \text { by }(3.8) .
\end{aligned}
$$

Hence we deduce that for all $x \in \mathbb{B}_{\delta}\left(x^{*}\right), Z_{1}(x) \in \mathbb{B}_{r_{0}}(0)$. Let $x^{\prime}, x^{\prime \prime} \in \mathbb{B}_{\delta}\left(x^{*}\right)$. This together with (3.7) (with $y_{1}=Z_{1}\left(x^{\prime}\right)$, and $\left.y_{2}=Z_{1}\left(x^{\prime \prime}\right)\right)$ implies that

$$
\begin{aligned}
e\left(\Phi_{1}\left(x^{\prime}\right)\right. & \left.\cap \mathbb{B}_{r_{x_{2}}}\left(x^{*}\right), \Phi_{1}\left(x^{\prime \prime}\right)\right) \leq e\left(\Phi_{1}\left(x^{\prime}\right) \cap \mathbb{B}_{\delta}\left(x^{*}\right), \Phi_{x}\left(x^{\prime \prime}\right)\right) \\
& =e\left(Q_{x^{*}}{ }^{-1}\left[Z_{1}\left(x^{\prime}\right)\right] \cap \mathbb{B}_{\delta}\left(x^{*}\right), Q_{x^{*}}{ }^{-1}\left[Z_{1}\left(x^{\prime \prime}\right)\right]\right) \\
& \leq M\left\|Z_{1}\left(x^{\prime}\right)-Z_{1}\left(x^{\prime \prime}\right)\right\| \\
& \leq M\left\|\left(\mathcal{D} f\left(x^{*}\right)-\mathcal{D} f\left(x_{1}\right)\right)\left(x^{\prime}-x^{\prime \prime}\right)+M\right\| g\left(x^{\prime}\right)-g\left(x^{\prime \prime}\right)-\left[x_{0}, x_{1} ; g\right]\left(x^{\prime}-x^{\prime \prime}\right) \| \\
& \leq M \epsilon\left\|x^{\prime}-x^{\prime \prime}\right\|+M\left\|\left[x^{\prime \prime}-x^{\prime} ; g\right]\left(x^{\prime}-x^{\prime \prime}\right)-\left[x_{0}, x_{1} ; g\right]\left(x^{\prime}-x^{\prime \prime}\right)\right\| \\
& \leq M \epsilon\left\|x^{\prime}-x^{\prime \prime}\right\|+M\left\|\left(\left[x^{\prime \prime}-x^{\prime} ; g\right]-\left[x_{0}, x_{1} ; g\right]\right)\left(x^{\prime}-x^{\prime \prime}\right)\right\| \\
& \leq M \epsilon\left\|x^{\prime}-x^{\prime \prime}\right\|+M\left\|\kappa\left(\left\|x^{\prime \prime}-x_{0}\right\|+\left\|x^{\prime}-x_{1}\right\|\right)\right\|\left\|x^{\prime}-x^{\prime \prime}\right\| \\
& \leq M \epsilon\left\|x^{\prime}-x^{\prime \prime}\right\|+M \kappa(2 \delta+2 \delta)\left\|x^{\prime}-x^{\prime \prime}\right\| \\
\leq & M(\epsilon+4 \kappa \delta)\left\|x^{\prime}-x^{\prime \prime}\right\| .
\end{aligned}
$$

Due to the relation $28 M \kappa \delta \leq 4-7 M \epsilon$ in (3.8), we obtain from (3.12) that

$$
e\left(\Phi_{1}\left(x^{\prime}\right) \cap \mathbb{B}_{r_{x_{2}}}\left(x^{*}\right), \Phi_{1}\left(x^{\prime \prime}\right)\right) \leq \frac{4}{7}\left\|x^{\prime}-x^{\prime \prime}\right\|=\lambda\left\|x^{\prime}-x^{\prime \prime}\right\|
$$

Thus assertion (b) of Lemma 1 is satisfied. This completes the proof of the Lemma.

Theorem 1. Let $x^{*}$ be a solution of (1.1). Suppose that assumptions (A0)-(A5) are satisfied. Let $C$ be defined in (3.4). Then for every $C$, there exists $\delta>0$ such that for every starting point $x_{0}, x_{1}, \in \mathbb{B}_{\delta}\left(x^{*}\right)$, there exists a sequence $\left\{x_{k}\right\}$ generated by (1.4) with initial point $x_{0}, x_{1}$ which converges to $x^{*}$ and satisfies the following inequality

$$
\left\|x_{k+1}-x^{*}\right\| \leq C\left\|x_{k}-x^{*}\right\| \quad \text { for each } k=1,2, \ldots
$$


Proof. By Lemma 2, for every $C$, there exists $\delta>0$ such that for each $x_{0}, x_{1} \in \mathbb{B}_{\delta}\left(x^{*}\right)$, there is $x_{2} \in \mathbb{B}_{\delta}\left(x^{*}\right)$ such that (3.5) and (3.6) hold. Let $x_{0}, x_{1} \in \mathbb{B}_{\delta}\left(x^{*}\right)$. It follows from Lemma 2 that there exists $x_{2} \in \mathbb{B}_{\delta}\left(x^{*}\right)$ such that

$$
0 \in f\left(x_{1}\right)+g\left(x_{1}\right)+\left(\mathcal{D} f\left(x_{1}\right)+\left[x_{0}, x_{1} ; g\right]\right)\left(x_{2}-x_{1}\right)+F\left(x_{2}\right)
$$

and

$$
\left\|x_{2}-x^{*}\right\| \leq r_{x_{2}} \leq C\left\|x_{1}-x^{*}\right\|
$$

and so (3.13) holds for $k=1$. We will proceed by induction. Now assume that $x_{0}, x_{1}, \ldots, x_{k}$ are generated by (1.4) satisfying (3.13). Then by Lemma 2, we can choose $x_{k+1} \in \mathbb{B}_{\delta}\left(x^{*}\right)$ such that

$$
0 \in f\left(x_{k}\right)+g\left(x_{k}\right)+\left(\mathcal{D} f\left(x_{k}\right)+\left[x_{k-1}, x_{k} ; g\right]\right)\left(x_{k+1}-x_{k}\right)+F\left(x_{k+1}\right)
$$

and

$$
\left\|x_{k+1}-x^{*}\right\| \leq r_{x_{2}} \leq C\left\|x_{k}-x^{*}\right\|,
$$

and so (3.13) holds for all $k \geq 1$. This completes the proof of the Theorem.

\subsection{Superlinear Convergence}

This subsection is devoted to study the superlinear convergence result of the Newton-like method (1.4). To do this, we will take the following assumptions:

(A6) $\mathcal{D} f$ is Lipschitz continuous in a neighbourhood $\Omega$ of $x^{*}$ with constant $L$ i.e. for every $x, y \in \Omega$, we have that

$$
\|\mathcal{D} f(x)-\mathcal{D} f(y)\|<L\|x-y\|
$$

(A7) $g$ admits first order divided difference i.e. there exists $\kappa>0$ such that, for all $x, y, x^{\prime}, y^{\prime} \in \Omega$,

$$
\left\|[x, y ; g]-\left[x^{\prime}, y^{\prime} ; g\right]\right\| \operatorname{leq\kappa }\left(\left\|x-x^{\prime}\right\|^{2}+\left\|y-y^{\prime}\right\|^{2}\right) \quad \text { with } x^{\prime} \neq x, y^{\prime} \neq y \text {. }
$$

Let $M, L$ and $\kappa$ be defined in (A3), (A6) and (A7) such that $3 M(L+8 \kappa)<1$. Let

$$
\text { Set } \gamma:=\frac{3 M(L+8 \kappa)}{2}
$$

Then we obtain that $\gamma<\frac{1}{2}$.

Lemma 3. Let $x^{*}$ be a solution of (1.1). Suppose that assumptions (A0)-(A3), (A6) and (A7) are hold.. Let $\gamma$ be defined by (3.14). Then for every such $\gamma$, there exists $\delta>0$ such that for every distinct starting point $x_{0}, x_{1} \in B_{\delta}\left(x^{*}\right)$, there exists a sequence $\left\{x_{2}\right\}$, defined by

$$
0 \in f\left(x_{1}\right)+g\left(x_{1}\right)+\left(\mathcal{D} f\left(x_{1}\right)+\left[x_{0}, x_{1} ; g\right]\right)\left(x_{2}-x_{1}\right)+F\left(x_{2}\right)
$$

and the map $\Phi_{1}$ has a fixed point $x_{2}$ in $B_{\delta}\left(x^{*}\right)$, which satisfies

$$
\left\|x_{2}-x^{*}\right\| \leq \gamma\left\|x_{1}-x^{*}\right\| \max \left\{\left\|x_{1}-x^{*}\right\|,\left\|x_{1}-x_{0}\right\|\right\}
$$

Proof. The assumption (A3) implies that the mapping $Q_{x^{*}}^{-1}$ is $M$-pseudo-Lipschitz around $\left(0, x^{*}\right)$. Hence there exists $r_{x^{*}}>0$ and $r_{0}>0$ such that

$$
e\left(Q_{x}^{*-1}\left(y_{1}\right) \cap \mathbb{B}_{r_{x^{*}}}\left(x^{*}\right), Q_{x^{*}}^{-1}\left(y_{2}\right)\right) \leq M\left\|y_{1}-y_{2}\right\| \text { for any } y_{1}, y_{2} \in \mathbb{B}_{r_{0}}(0) .
$$

Let $\delta>0$ be such that

$$
\delta \leq \max \left\{r_{x^{*}}, \sqrt{\frac{2 r_{0}}{5 L+32 \kappa}}, \frac{2}{3 M(5 L+8 \kappa)}, 1\right\} .
$$

Fix $x_{0}, x_{1} \in \mathbb{B}_{\delta}\left(x^{*}\right)$ such that $x_{0} \neq x_{1} \neq x^{*}$, and define

$$
r_{x_{2}}=\gamma\left\|x_{1}-x^{*}\right\| \max \left\{\left\|x_{1}-x^{*}\right\|,\left\|x_{1}-x_{0}\right\|\right\} .
$$


This implies that $r_{x_{2}} \leq \gamma \delta . \delta \leq \gamma \delta \leq \delta$ and hence $r_{x_{2}} \leq \delta \leq r_{x^{*}}$. We will apply Lemma 1 to the map $\Phi_{1}$ with $\eta_{0}:=x^{*}$ and $r:=r_{x_{2}}$ and $\lambda:=\frac{2}{3}$ to conclude that there exists a fixed point $x_{2} \in \mathbb{B}_{r_{x_{2}}}\left(x^{*}\right)$ such that $x_{2} \in \Phi_{1}\left(x_{2}\right)$, that is, $Z_{1}\left(x_{2}\right) \in Q_{x^{*}}{ }^{-1}\left(x_{2}\right)$, which implies that

$$
0 \in f\left(x_{1}\right)+g\left(x_{1}\right)+\left(\mathcal{D} f\left(x_{1}\right)+\left[x_{0}, x_{1} ; g\right]\right)\left(x_{2}-x_{1}\right)+F\left(x_{2}\right),
$$

Furthermore, $x_{2} \in \mathbb{B}_{r_{x_{2}}}\left(x^{*}\right) \subseteq \mathbb{B}_{\delta}\left(x^{*}\right)$ and so

$$
\left\|x_{2}-x^{*}\right\| \leq r_{x_{2}} \leq \gamma\left\|x_{1}-x^{*}\right\| \max \left\{\left\|x_{1}-x^{*}\right\|,\left\|x_{1}-x_{0}\right\|\right\} .
$$

Thus, to complete the proof, it is sufficient to show that Lemma 1 is applicable for the map $\Phi_{1}$ with $\eta_{0}:=x^{*}$ and $r:=r_{x_{2}}$ and $\lambda:=\frac{2}{3}$. To do this, it remains to prove that both assertions (a) and (b) of Lemma 1 hold. It is obvious that $x^{*} \in Q_{x^{*}}{ }^{-1}(0) \cap \mathbb{B}_{r_{x_{2}}}\left(x^{*}\right)$. According to the definition of the excess $e$, we have

$$
\operatorname{dist}\left(x^{*}, \Phi_{1}\left(x^{*}\right)\right) \leq e\left(Q_{x^{*}}{ }^{-1}(0) \cap \mathbb{B}_{r_{x_{2}}}\left(x^{*}\right), \Phi_{1}\left(x^{*}\right)\right) .
$$

Moreover, for all $x_{0}, x_{1} \in \mathbb{B}_{r_{x_{2}}}\left(x^{*}\right)$ such that $x_{0}, x_{1}$ and $x^{*}$ are distinct, we have from (3.2) that

$$
\begin{aligned}
\left\|Z_{1}\left(x^{*}\right)\right\|= & \| f\left(x^{*}\right)+g\left(x^{*}\right)-f\left(x_{1}\right)-g\left(x_{1}\right)-\left(\mathcal{D} f\left(x_{1}\right)+\left[x_{0}, x_{1} ; g\right]\right)\left(x^{*}-x_{1}\right) \\
\leq & \left\|f\left(x^{*}\right)-f\left(x_{1}\right)-\mathcal{D} f\left(x_{1}\right)\left(x^{*}-x_{1}\right)\right\|+\left\|g\left(x^{*}\right)-g\left(x_{1}\right)-\left[x_{0}, x_{1} ; g\right]\left(x^{*}-x_{1}\right)\right\| \\
& \quad[\text { By using definition }(6)] . \\
& =\left\|f\left(x^{*}\right)-f\left(x_{1}\right)-\mathcal{D} f\left(x_{1}\right)\left(x^{*}-x_{1}\right)\right\|+\left\|\left[x_{1}, x^{*} ; g\right]\left(x^{*}-x_{1}\right)-\left[x_{0}, x_{1} ; g\right]\left(x^{*}-x_{1}\right)\right\| \\
& =\left\|f\left(x^{*}\right)-f\left(x_{1}\right)-\mathcal{D} f\left(x_{1}\right)\left(x^{*}-x_{1}\right)\right\|+\left\|\left(\left[x_{1}, x^{*} ; g\right]-\left[x_{0}, x_{1} ; g\right]\right)\left(x^{*}-x_{1}\right)\right\|
\end{aligned}
$$

Since $f\left(x^{*}\right)-f\left(x_{1}\right)-\mathcal{D} f\left(x_{1}\right)\left(x^{*}-x_{1}\right)=\int_{0}^{1}\left[\mathcal{D} f\left(x_{1}+t\left(x^{*}-x_{1}\right)\right)-\mathcal{D} f\left(x_{1}\right)\right]\left(x^{*}-x_{1}\right) d t$,

$$
\begin{aligned}
\left\|Z_{1}\left(x^{*}\right)\right\| \leq & \int_{0}^{1}\left[\mathcal{D} f\left(x_{1}+t\left(x^{*}-x_{1}\right)\right)-\mathcal{D} f\left(x_{1}\right)\right]\left(x^{*}-x_{1}\right) d t+\left\|\left[x_{1}, x^{*} ; g\right]-\left[x_{0}, x_{1} ; g\right]\right\|\left\|x^{*}-x_{1}\right\| \\
\leq & \int_{0}^{1} L\left\|\left(x_{1}+t\left(x^{*}-x_{1}\right)-x_{1}\right)\right\|\left\|x^{*}-x_{1}\right\| d t+\kappa\left(\left\|x_{1}-x_{0}\right\|^{2}+\left\|x^{*}-x_{0}\right\|^{2}\right)\left\|x^{*}-x_{1}\right\| \\
& {[\text { By using assumption (A6) and (A7)] }} \\
& \int_{0}^{1} L\left\|t\left(x^{*}-x_{1}\right)\right\| d t\left\|x^{*}-x_{1}\right\|+\kappa\left(\left\|x_{1}-x_{0}\right\|^{2}+\left\|x^{*}-x_{0}\right\|^{2}\right)\left\|x^{*}-x_{1}\right\| \\
& \leq \frac{L}{2}\left\|x^{*}-x_{1}\right\|^{2}+2 \kappa\left\|x_{1}-x_{0}\right\|^{2}\left\|x^{*}-x_{1}\right\| \\
& \leq \frac{L}{2}\left\|x^{*}-x_{1}\right\|^{2}+2 \kappa .2 \delta\left\|x_{1}-x_{0}\right\|\left\|x^{*}-x_{1}\right\| \\
& \leq \frac{L}{2}\left\|x^{*}-x_{1}\right\|^{2}+4 \kappa\left\|x_{1}-x_{0}\right\|\left\|x^{*}-x_{1}\right\|, \text { since } \delta \leq 1 \\
& \leq \frac{L}{2} \delta^{2}+8 \kappa . \delta . \delta=\left(\frac{L}{2}+8 \kappa\right) \delta^{2} \\
& <r_{0} \text { by }(3.18) .
\end{aligned}
$$

This together with (3.17) and (3.19) (with $y_{1}=0$ and $y_{2}=Z_{1}\left(x^{*}\right)$ ) implies that

$$
\begin{aligned}
\operatorname{dist}\left(x^{*}, \Phi_{1}\left(x^{*}\right)\right) & \leq M\left\|y_{1}-y_{2}\right\| \leq M\left\|Z_{1}\left(x^{*}\right)\right\| \\
& \leq M\left(\frac{L}{2}\left\|x^{*}-x_{1}\right\|^{2}+4 \kappa\left\|x_{1}-x_{0}\right\|\left\|x^{*}-x_{1}\right\|\right) \text { by using (3.20) } \\
& \leq M\left(\frac{L}{2}+4 \kappa\right)\left\|x_{1}-x^{*}\right\| \max \left\{\left\|x_{1}-x^{*}\right\|,\left\|x_{1}-x_{0}\right\|\right\} \\
& =\left(1-\frac{2}{3}\right) \frac{3 M(L+8 \kappa)}{2}\left\|x_{1}-x^{*}\right\| \max \left\{\left\|x_{1}-x^{*}\right\|,\left\|x_{1}-x_{0}\right\|\right\} . \\
& =\left(1-\frac{2}{3}\right) r_{x_{2}}=r(1-\lambda) .
\end{aligned}
$$


Hence assertion (a) of Lemma 1 is satisfied.

Now, we show that assertion (b) of Lemma 1 is also satisfied. Let $x \in \mathbb{B}_{r_{x_{2}}}\left(x^{*}\right) \subseteq \mathbb{B}_{\delta}\left(x^{*}\right)$. Then

$$
\begin{aligned}
\left\|Z_{1}(x)\right\|= & \left\|f\left(x^{*}\right)+g(x)-\mathcal{D} f\left(x^{*}\right)\left(x^{*}-x\right)-f\left(x_{1}\right)-g\left(x_{1}\right)-\left(\mathcal{D} f\left(x_{1}\right)+\left[x_{0}, x_{1} ; g\right]\right)\left(x^{*}-x_{1}\right)\right\| \\
= & \| f\left(x^{*}\right)-f(x)+f(x)-f\left(x_{1}\right)-\mathcal{D} f\left(x^{*}\right)\left(x^{*}-x\right)+g(x)-g\left(x_{1}\right) \\
& -\left(\mathcal{D} f\left(x_{1}\right)\left(x-x_{1}\right)-\left[x_{0}, x_{1} ; g\right]\right)\left(x-x_{1}\right) \| \\
\leq & \left\|f\left(x^{*}\right)-f(x)-\mathcal{D} f\left(x^{*}\right)\left(x^{*}-x\right)\right\|+\left\|f(x)-f\left(x_{1}\right)-\mathcal{D} f\left(x_{1}\right)\left(x-x_{1}\right)\right\| \\
& +\left\|g(x)-g\left(x_{1}\right)-\left[x_{0}, x_{1} ; g\right]\left(x-x_{1}\right)\right\| \\
\leq & \frac{L}{2}\left\|x-x^{*}\right\|^{2}+\frac{L}{2}\left\|x-x_{1}\right\|^{2}+\left\|\left[x_{1}, x ; g\right]\left(x-x_{1}\right)-\left[x_{0}, x_{1} ; g\right]\left(x-x_{1}\right)\right\| \\
= & \frac{L}{2}\left\|x-x^{*}\right\|^{2}+\frac{L}{2}\left\|x-x_{1}\right\|^{2}+\left\|\left[x_{1}, x ; g\right]-\left[x_{0}, x_{1} ; g\right]\right\|\left\|x-x_{1}\right\| \\
\leq & \frac{L}{2}\left\|x-x^{*}\right\|^{2}+\frac{L}{2}\left\|x-x_{1}\right\|^{2}+\kappa\left(\left\|x_{1}-x_{0}\right\|^{2}+\left\|x-x_{1}\right\|^{2}\left\|x-x_{1}\right\|\right. \\
\leq & \frac{L}{2} \delta^{2}+\frac{L}{2}(2 \delta)^{2}+\kappa\left((2 \delta)^{2}+(2 \delta)^{2}\right) \cdot 2 \delta \\
= & \frac{L}{2} \delta^{2}+2 L \delta^{2}+16 \kappa \delta^{3} \leq \frac{L}{2} \delta^{2}+2 L \delta^{2}+16 \kappa \delta^{2}, \text { since } \delta \leq 1 \\
= & \left(\frac{5 L}{2}+16 \kappa\right) \delta^{2}<r_{0}, \text { by }(3.18) .
\end{aligned}
$$

Hence we deduce that for all $x \in \mathbb{B}_{\delta}\left(x^{*}\right), Z_{1}(x) \in \mathbb{B}_{r_{0}}(0)$. Let $x^{\prime}, x^{\prime \prime} \in \mathbb{B}_{r_{x_{2}}}\left(x^{*}\right)$. This together with (3.17) (with $y_{1}=Z_{1}\left(x^{\prime}\right)$, and $\left.y_{2}=Z_{1}\left(x^{\prime \prime}\right)\right)$ implies that

$$
\begin{aligned}
e\left(\Phi_{1}\left(x^{\prime}\right)\right. & \left.\cap \mathbb{B}_{r_{x_{2}}}\left(x^{*}\right), \Phi_{1}\left(x^{\prime \prime}\right)\right) \leq e\left(\Phi_{1}\left(x^{\prime}\right) \cap \mathbb{B}_{\delta}\left(x^{*}\right), \Phi_{x}\left(x^{\prime \prime}\right)\right. \\
& =e\left(Q_{x^{*}}^{-1}\left[Z_{1}\left(x^{\prime}\right)\right] \cap \mathbb{B}_{\delta}\left(x^{*}\right), Q_{x^{*}}^{-1}\left[Z_{1}\left(x^{\prime \prime}\right)\right]\right) \\
\leq & M\left\|Z_{1}\left(x^{\prime}\right)-Z_{1}\left(x^{\prime \prime}\right)\right\| \\
\leq & M\left\|\left(\mathcal{D} f\left(x^{*}\right)-\mathcal{D} f\left(x_{1}\right)\right)\left(x^{\prime}-x^{\prime \prime}\right)\right\|+M\left\|g\left(x^{\prime}\right)-g\left(x^{\prime \prime}\right)-\left[x_{0}, x_{1} ; g\right]\left(x^{\prime}-x^{\prime \prime}\right)\right\| \\
\leq & M L\left\|x^{*}-x_{1}\right\|\left\|x^{\prime}-x^{\prime \prime}\right\|+M\left\|\left[x^{\prime \prime}, x^{\prime} ; g\right]\left(x^{\prime}-x^{\prime \prime}\right)-\left[x_{0}, x_{1} ; g\right]\left(x^{\prime}-x^{\prime \prime}\right)\right\| \\
\leq & M L\left\|x^{*}-x_{1}\right\|\left\|x^{\prime}-x^{\prime \prime}\right\|+M\left\|\left(\left[x^{\prime \prime}, x^{\prime} ; g\right]-\left[x_{0}, x_{1} ; g\right]\right)\left(x^{\prime}-x^{\prime \prime}\right)\right\| \\
\leq & M L\left\|x^{*}-x_{1}\right\|\left\|x^{\prime}-x^{\prime \prime}\right\|+M \kappa\left(\left\|x^{\prime \prime}-x_{0}\right\|^{2}+\left\|x^{\prime}-x_{1}\right\|^{2}\right)\left\|x^{\prime}-x^{\prime \prime}\right\| \\
\leq & M L \delta\left\|x^{\prime}-x^{\prime \prime}\right\|+M \kappa\left((2 \delta)^{2}+(2 \delta)^{2}\right)\left\|x^{\prime}-x^{\prime \prime}\right\| \\
\leq & M L \delta\left\|x^{\prime}-x^{\prime \prime}\right\|+M \kappa 8 \delta^{2}\left\|x^{\prime}-x^{\prime \prime}\right\| \\
\leq & M(L+8 \kappa) \delta\left\|x^{\prime}-x^{\prime \prime}\right\|
\end{aligned}
$$

Now using the relation $3 M(L+8 \kappa) \delta \leq 2$ from(3.18) in (3.21), we have

$$
e\left(\Phi_{1}\left(x^{\prime}\right) \cap \mathbb{B}_{r_{x_{2}}}\left(x^{*}\right), \Phi_{1}\left(x^{\prime \prime}\right)\right) \leq \frac{2}{3}\left\|\left(x^{\prime}-x^{\prime \prime}\right)\right\|=\lambda\left\|\left(x^{\prime}-x^{\prime \prime}\right)\right\| .
$$

Thus assertion (b) of Lemma 1 is satisfied. This completes the proof of the Lemma.

Theorem 2. Let $x^{*}$ be a solution of (1.1). Suppose that assumptions (A0)-(A3),(A6) and (A7) are satisfied. Let $\gamma$ be defined in (3.14). Then for every $\gamma$, there exists $\delta>0$ such that for every starting point $x_{0}, x_{1}, \in \mathbb{B}_{\delta}\left(x^{*}\right)$, there exists a sequence $\left\{x_{k}\right\}$ generated by (1.4) with initial point $x_{0}, x_{1}$ which converges to $x^{*}$ and satisfies that

$$
\left\|x_{k+1}-x^{*}\right\| \leq \gamma\left\|x_{k}-x^{*}\right\| \max \left\{\left\|x_{k}-x^{*}\right\|,\left\|x_{k}-x_{k-1}\right\|\right\} \text { for each } k=1,2, \ldots
$$

Proof. By Lemma 3, for every $\gamma$, there exists $\delta>0$ such that for each $x_{0}, x_{1} \in \mathbb{B}_{\delta}\left(x^{*}\right)$, there is $x_{2} \in \mathbb{B}_{\delta}\left(x^{*}\right)$, such that (3.15) and 3.16) hold. Let $x_{0}, x_{1} \in \mathbb{B}_{\delta}\left(x^{*}\right)$. It follows from Lemma 3 that there exists $x_{2} \in \mathbb{B}_{\delta}\left(x^{*}\right)$ such that

$$
0 \in f\left(x_{1}\right)+g\left(x_{1}\right)+\left(\mathcal{D} f\left(x_{1}\right)+\left[x_{0}, x_{1} ; g\right]\right)\left(x_{2}-x_{1}\right)+F\left(x_{2}\right)
$$

and

$$
\left\|x_{2}-x^{*}\right\| \leq r_{x_{2}} \leq \gamma\left\|x_{1}-x^{*}\right\| \max \left\{\left\|x_{1}-x^{*}\right\|,\left\|x_{1}-x_{0}\right\|\right\}
$$


and so (3.22) holds for $k=1$. We will proceed by induction. Now assume that $x_{0}, x_{1}, \ldots, x_{k}$ are generated by (1.4) satisfying (3.22). Then by Lemma 3 , we can choose $x_{k+1} \in \mathbb{B}_{\delta}\left(x^{*}\right)$ such that

$$
0 \in f\left(x_{k}\right)+g\left(x_{k}\right)+\left(\mathcal{D} f\left(x_{k}\right)+\left[x_{k-1}, x_{k} ; g\right]\right)\left(x_{k+1}-x_{k}\right)+F\left(x_{k+1}\right)
$$

and

$$
\left\|x_{k+1}-x^{*}\right\| \leq r_{x_{k+1}} \leq \gamma\left\|x_{k}-x^{*}\right\| \max \left\{\left\|x_{k}-x^{*}\right\|,\left\|x_{k}-x_{k-1}\right\|\right\}
$$

and so (3.22) holds for all $k \geq 1$. This completes the proof of the Theorem.

\section{Concluding Remark}

We have established local convergence results of the Newton-like method for approximating the solution of the variational inclusion (1.1) under the assumptions that $Q_{x^{*}}^{-1}$ is pseudo-Lipschitz and $\mathcal{D} f$ is continuous, Lipschitz continuous and $\mathrm{g}$ is admissible for first order divided difference. More clearly, we have shown that the Newton-like method defined by (1.4) converges linearly and superlinearly to the solution of (1.1) if $\mathcal{D} f$ is continuous and Lipschitz continuous, respectively, together with a divided difference admissible function $g$. This study improves and extends the results corresponding to [11].

\section{Acknowledgments}

The research work of first author is supported by University Grant Commission, Bangladesh, grant No. 1.157 and 5340/2016 while the research work of second author is financed by Faculty of Science, University of Rajshahi, Bangladesh, grant No. 1148/5/52/R.U./Science-02/19-20.

\section{References}

[1] Aubin, J. P., (1984). Lipschitz behavior of solutions to convex maximization problems, Mathematics of Operations Research 9, p. 87.

[2] Cătinas, E., (1994). On some iterative methods for solving nonlinear equations, Rev. Anal. Numér. Theor. Approx. 23, p. 17.

[3] Dontchev, A.L., (1996). Local convergence of the Newton method for Generalized Equations, C. R. Acad. Sci. Paris, Ser. I. 322, p. 327.

[4] Dontchev, A. L., (1996). Local analysis of a Newton-type method based on partial linearization, Lectures in Applied Mathematics 32, p. 295.

[5] Dontchev, A. L., (1996). Uniform convergence of the Newton method for Aubin continuous maps, Serdica Math. J. 22, p. 385.

[6] Dontchev, A. L. and Hager, W. W., (1994). An inverse mapping theorem for set-valued maps, Proc. Amer. Math. Soc. 121, p. 481.

[7] Dontchev, A.L. and Rockafellar R.T., (2004). Regularity and conditioning of solution mappings in variational analysis, Set-valued Anal. 12(1), p. 79.

[8] Ferris, M.C. and Pang, J.S., (1997). Engineering and economic applications of complementarily problems, SIAM Rev. 39, p. 669.

[9] Geoffroy, M. H., Hilout, S. and Pietrus, A., (2003). Acceleration of convergence in Dontchev's iterative methods for solving generalized equations, Serdica Math. J. 29, p. 45.

[10] Geoffroy, M.H., and Pietrus, A., (2005). A general iterative procedure for solving nonsmooth generalized equations, Comput. Optim. Appl. 31(1), p. 57.

[11] Geoffroy, M.H. and Pietrus, A., (2004). Local convergence of some iterative methods for generalized equations, J. Math. Anal. Appl. 290, p. 497. 
[12] He. J.S., Wang, J.H. and Li, C., (2007). Newton's method for undetermined systems of equations under the modified $\gamma$-condition, Numer. Funct. Anal. Optim. 28, p. 663.

[13] Jean-Alexis, C. and Pietrus, A., (2008). On the convergence of some methods for variational inclusions, Rev. R. Acad. Cien. serie A. Mat. 102(2), p. 355.

[14] Lawrence, C. E.: Partial Differential Equation (second edition), American Mathematical Society, 1988.

[15] Marinov, R.T., (2009). Convergence of the method of chords for solving generalized Equations, Rendiconti del Circolo Matematico di Palermo 58, p. 11.

[16] Pietrus, A., (2000). Does Newton's method for set-valued maps converge uniformly in mild di?erentiability context? Rev. Columbiana Mat. 34, p. 49.

[17] Pietrus, A., (2000). Generalized equations under mild differentiability conditions, Rev. S. A. Acad. Cienc. Exact. Fis. Nat. 94(1), p. 15.

[18] Pietrus, A. and Hilout, S., (2006). A semi-local convergence of a secant-type method for solving generalized equations, Positivity 10(4), p. 693.

[19] Rashid, M.H., (2014). On the convergence of extended Newton-type method for solving variational inclusions, Cogent Mathematics 1(1), p. 1.

[20] Rashid, M.H., (2014). Convergence analysis of gauss-type proximal point method for variational inequalities, Open Science Journal of Mathematics and Application 2(1), p. 5.

[21] Rashid, M. H., (2017). Extended Newton-type Method and its Convergence Analysis for Nonsmooth Generalized Equations, Journal of Fixed Point Theory and Applications, 19, p. 1295.

[22] Rashid, M. H., (2018). Convergence Analysis of a Variant of Newton-type Method for Generalized Equations, International Journal of Computer Mathematics, 95(3), p. 584.

[23] Rashid, M. H., Yu, S. H., Li, C. and Wu, S. Y., (2013). Convergence Analysis of the Gauss-Newton-type Method for Lipschitz-like Mappings, J. Optim. Theory Appl. 158(1), p. 216.

[24] Rashid, M.H., Wang, J.H. and Li, C., (2012). Convergence Analysis of a method for variational inclusions, Applicable Analysis 91(10), p. 1943.

[25] Robinson, S.M., (1979). Generalized equations and their solutions, Part I, basic theory, Math. Program. Stud. 10, p. 128.

[26] Robinson, S.M., (1982). Generalized equations and their solutions, part II: application to nonlinear programming, Math. Program. Stud. 19, p. 200. 